\section{Bromoderma tuberosum}

Zusammenfassung. Unter antikonvulsiver Therapie einer generalisierten Epilepsie mit Kaliumbromid entwickelte sich bei einem 6jährigen Jungen ein ausgeprägtes Bromoderma tuberosum des Gesichtes. Nach Abbruch der Kaliumbromidtherapie heilte der Befund innerhalb von 6 Wochen ab, wobei in umschriebenen Arealen zarte Narbenbildungen beobachtet werden mussten. Nachdem Kaliumbromid in den letzten Jahren wieder verstärkt zur Behandlung kindlicher Epilepsieformen eingesetzt wird, muss möglicherweise auch mit einer Zunahme der Zahl bromidinduzierter Dermatosen gerechnet werden.

Tuberous bromoderma. A six-year old boy taking potassium bromide because of generalized seizures displayed an extensive tuberous bromoderma of the face. Discontinuation of this anticonvulsant therapy resulted in healing of all but some skin lesions leaving discrete scars in some areas. Following the recent revival of potassium bromide for treating infant-type seizures there will probably be an increased incidence of bromide induced dermatoses.

\section{Einleitung}

Die Bromide als Salze der Bromwasserstoffsäure sowie die Bromureide als Bromharnstoffderivate fanden in der Vergangenheit zur Therapie der Epilepsien oder als Schlaf- und Beruhigungsmittel weite Verbreitung. Die meisten der ursprünglich frei verkäuflichen Bromureide wurden jedoch in den 80er Jahren vom Markt genommen, nachdem sie, als Hypnotika eingesetzt, schnell zu Abhängigkeiten führten oder gehäuft in suizidaler Absicht eingenommen wurden [10]. In der Zwischenzeit ist die Herstellung bromureidhaltiger Arzneimittel vollständig eingestellt worden, so dass auch mit den Nebenwirkungen dieser Substanzgruppe nicht mehr gerechnet werden muss.

Bei den Bromiden wurde das Kaliumbromid als Antikonvulsivum erstmals 1857 zur Therapie der Epilepsien eingesetzt. Als wichtigster Vertreter der Bromide verlor aber auch das Kaliumbromid bereits in den 50er Jahren an Bedeutung, nach-

Akt Dermatol 2001; 27: 97-99

(c) Georg Thieme Verlag Stuttgart · New York ISSN 0340-2541

\author{
G. Wagner \\ Hautklinik Zentralkrankenhaus Bremerhaven
}

dem neue Antiepileptika entwickelt worden waren, die sich unter anderem auch durch geringere Nebenwirkungen auszeichneten. Nachdem vor etwa 10 Jahren über erste Behandlungserfolge mit Kaliumbromid bei sonst therapieresistenten Epilepsien berichtet wurde, ist das Kaliumbromid inzwischen wieder fester Bestandteil der neurologischen Therapie, was insbesondere für die generalisierten kindlichen Epilepsieformen gilt [3]. Somit besteht heute durchaus wieder die Möglichkeit, mit bromidinduzierten Dermatosen konfrontiert zu werden, die fast in Vergessenheit geraten waren.

\section{Kasuistik}

\section{Anamnese}

Der jetzt 6jährige, mehrfachbehinderte Junge erkrankte im 1. Lebensjahr infolge eines frühkindlichen Hirnschadens an einer spastischen Zerebralparese in Kombination mit einer generalisierten Epilepsie. Die antikonvulsive Therapie war über Jahre mit Carbamazepin (Timonil ${ }^{\circledR}$ ) und Lamotrigen (Lamictal ${ }^{\circledR}$ ) durchgeführt worden. Trotz dieser Behandlung kam es immer häufiger zu Krampfanfällen, so dass im September 1999 erstmals Kaliumbromid (DIBRO-Be mono ${ }^{\circledR}$ ) in einer Tagesdosierung von $425 \mathrm{mg}$ verordnet wurde. Diese Maßnahme führte zu einer Reduktion der Zahl der Krampfanfälle. Im Februar 2000 bemerkte die Mutter eine anfänglich sehr umschriebene pustulöse Hautveränderung an der linken Wange, die in den folgenden Wochen kontinuierlich an Größe zunahm. Gleichzeitig traten auch in anderen Lokalisationen des Gesichtes weitere Morphen auf. Unter der Verdachtsdiagnose einer vegetierenden Pyodermie wurden verschiedene orale Antibiotika verordnet, ohne dass dies einen feststellbaren Einfluss auf die Dermatose zeigte. Im Mai 2000 erfolgte die Vorstellung in der Hautklinik Bremerhaven.

\section{Dermatologischer Befund}

An den Wangen sowie im Bereich der Schläfenregionen bds. fingernagel- bis münzgroße, auch konfluierende, teilweise scharf begrenzte, flach erhabene bis plateauförmig aufgeworfene, rötlich-bräunlich tingierte Tumoren von relativ weicher Konsistenz. An der Oberfläche gelblich-bräunliche Krusten, leicht blutende Erosionen und vereinzelt bis linsengroße Pusteln (Abb. 1 -3). 


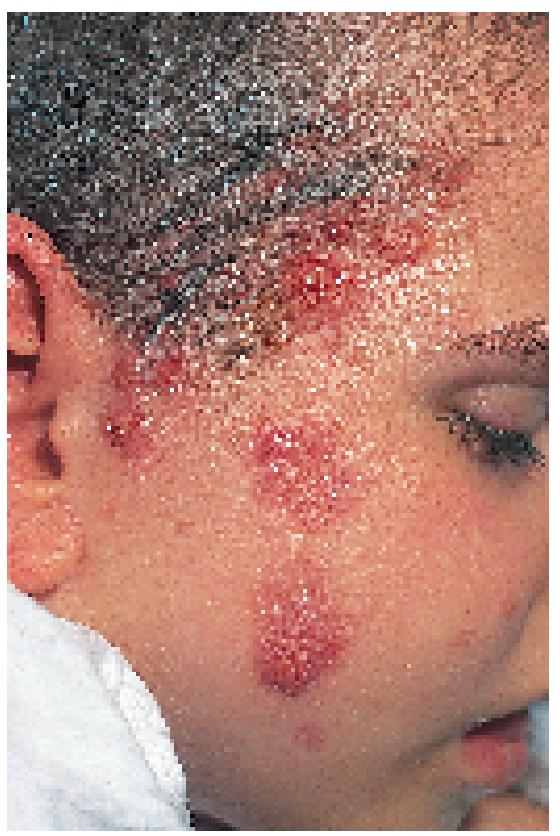

Abb. 1 Bromoderma tuberosum.

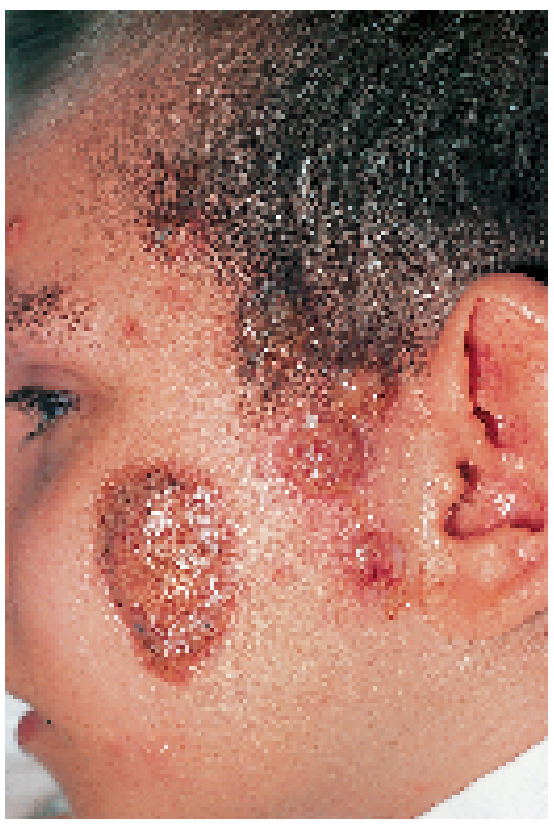

Abb. 3 Ältester Herd Wange links.

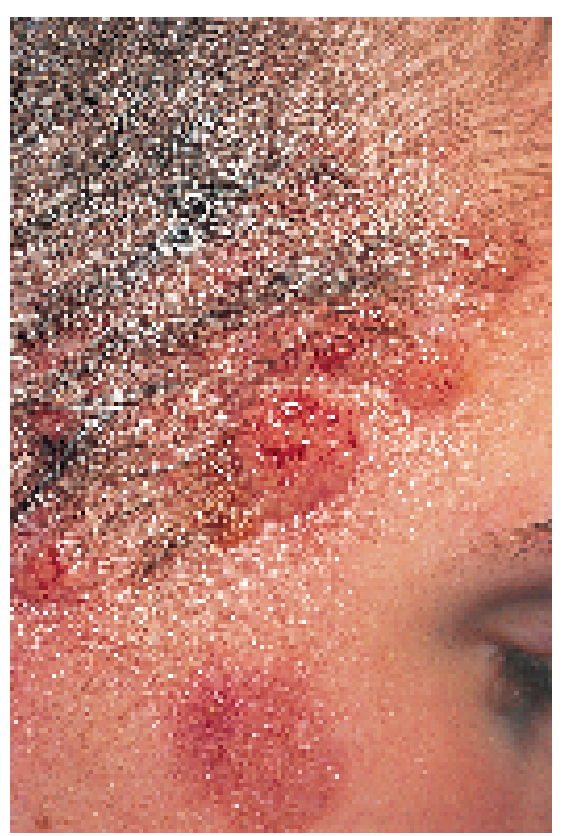

Abb. 2 Detailaufnahme.

Befunde diagnostischer Untersuchungen

Laborbefunde

Bromidspiegel im Serum $167 \mathrm{mg} / \mathrm{dl}$ (therapeutischer Bereich 96-144 mg/dl). Unauffällige Befunde: BB u. Diff. BB, BSG u. CRP, Transaminasen, Bilirubin, nierenpflichtige Substanzen, Elektrolyte und Eiweißelektrophorese.

\section{Mikrobiologische Befunde}

Nachweis von Staph. aureus und wenigen Kolonien Candida albicans.

\section{Therapie und Verlauf}

Die Behandlung mit Kaliumbromid wurde abgesetzt. Gleichzeitig erfolgte eine Steigerung der täglichen Dosierungen von Carbamazepin und Lamotrigen. Eine erneute Zunahme der Anfallshäufigkeit konnte unter diesem Behandlungsschema nicht beobachtet werden. Die dermatologische Therapie wurde ausschließlich lokal mit einer Erythromycin-Rezeptur durchgeführt. Nach 6 Wochen waren alle Befunde bis auf postinflammatorische Pigmentierungen und einzelne zarte Narben vollständig abgeheilt.

\section{Diskussion}

Zu den bromidinduzierten Dermatosen zählen das Bromexanthem, die Bromakne, die Brompannikulitis und das Bromoderma tuberosum. Dabei wird das Bromexanthem als polymorphes Arzneimittelexanthem beschrieben, bei dem der klinische Befund von Patient zu Patient deutlich variieren kann. Neben urtikariellen, roseoliformen und bullösen Exanthemen werden auch EEM-artige Manifestationen beobachtet [4]. Die Polymorphie und das Fehlen spezifischer oder typischer klinischer Merkmale erschweren bei den Bromexanthemen sowohl deren Diagnose als auch deren ätiologische Einordnung. Dies gilt in besonderer Weise für Epilepsiepatienten, die häufig verschiedene Antikonvulsiva gleichzeitig einnehmen müssen. Auch bei der Bromakne besteht das Problem der fehlenden richtungweisenden Befunde, die als Hinweis auf die auslösende Ursache dienen könnten. Bisweilen ist die Bromakne allerdings gekennzeichnet durch eine ausgeprägt entzündliche Komponente der einzelnen Morphen in Form größerer Papeln, Papulopusteln und plattenartiger Infiltrationen. Entsteht eine Akne bei bekannter Bromidanamnese jenseits des typischen 
Aknealters und möglicherweise auch außerhalb typischer Lokalisationen, so sind dies ebenfalls Hinweise für eine bromidinduzierte Auslösung der Akne. Grundsätzlich ist es möglich, dass eine Akne unter dem Einfluss der Bromide erstmals auftritt oder eine bereits bestehende Akne eine deutliche Befundverschlechterung erfährt [9].

Die Brompannikulitis zählt zu den selteneren Nebenwirkungen einer Bromidtherapie. Typischerweise treten erst nach längerer Einnahme von Kaliumbromid schmerzhafte Knoten an den Extremitäten auf, die anfänglich dem klinischen Bild eines Erythema nodosum entsprechen. Die Knoten können später jedoch einschmelzen und ulzerieren. Darüber hinaus ist die dermatologische Symptomatik von einer häufig deutlichen Paraklinik begleitet mit schwerem allgemeinen Krankheitsgefühl, hohem Fieber, Myalgien, abdominellen Krämpfen und Diarrhöen. Nach Abbruch der Bromidtherapie kommt es innerhalb kürzester Zeit zur Rückbildung der Symptome [1,2].

Im Gegensatz zu den bisher genannten bromidinduzierten Dermatosen zeichnet sich das Bromoderma tuberosum in seinem gesamten Verlauf durch klinisch charakteristische Merkmale aus, bei deren Kenntnis die Diagnose trotz des seltenen Vorkommens der Dermatose im Allgemeinen keine wesentlichen Schwierigkeiten bereiten dürfte. Das Bromoderma tuberosum besteht aus einzelnen oder häufiger multiplen, fingernagel- bis handtellergroßen, plaqueförmig erhabenen Tumoren. Besonders typisch sind deren scharfe Begrenzung, eine weiche, schwammartige Konsistenz und eine papillomatöse oder nässend-erosive Oberfläche mit Krusten und Pusteln. Frisch entstandene Läsionen zeigen einen rötlich-bräunlichen Farbton, während sich ältere Befunde durch eine dunkelbraune bis fast schwarze Pigmentierung auszeichnen können. Die einzelnen Knoten sind schmerzlos oder außerordentlich druckschmerzhaft. Zu den Prädilektionsstellen zählen bei Kindern besonders das Gesicht sowie bei Erwachsenen zusätzlich auch die unteren Extremitäten [4, 7, 8, 11,12\}. Die Pathogenese des Bromoderma tuberosum ist bis heute nicht geklärt. Dabei scheint die Induktion der entzündlichen Reaktion innerhalb der Epidermis dosisabhängig zu sein. Durch die lange Halbwertszeit der Bromide von etwa 12 Tagen ist eine kumulative Anreicherung im Organismus möglich [10]. Tatsächlich sind die Bromidspiegel im Serum bei den Patienten mit einem Bromoderma tuberosum in der Regel über den therapeutischen Bereich hinaus erhöht $[8,12]$. Die Elimination der Bromide erfolgt hauptsächlich durch die Nieren, jedoch wird ein geringer Anteil auch durch den Speichel, den Schweiß und die Talgdrüsen ausgeschieden [3]. Nach der von Kimmig propagierten Abspaltungstheorie ist das Bromoderma tuberosum das Ergebnis einer irritativ-toxischen Reaktion innerhalb der Epidermis, verursacht durch die Abspaltung gewebeaggressiver Bromionen aus den Bromiden [6].

Zur Behandlung des Bromoderma tuberosum wird üblicherweise der Abbruch der Bromidtherapie empfohlen. Allerdings sind vereinzelt auch Abheilungen unter alleiniger Dosisreduktion beobachtet worden [8]. Ein weiterer therapeutischer Ansatz ist die forcierte Diurese der Bromide durch Verordnung von Diuretika oder durch die Gabe von $\mathrm{NaCl}$, wobei das Chlorid mit dem Bromid um die tubuläre Rückresorption konkurriert $[3,5,12]$. Des Weiteren wurde vereinzelt auch über erfolgreiche Therapieversuche mit lokal oder oral verordneten Glukokortikoiden berichtet $[7,11]$.

\section{Literatur}

${ }^{1}$ Bork K. Arzneimittelnebenwirkungen an der Haut. 2. Aufl. Stuttgart: Schattenauer, 1999

2 Diener W, Kruse R, Berg P. Halogenpanniculitis auf Kaliumbromid. Monatsschr Kinderheilkd 1993; 141: 705-707

${ }^{3}$ Diener W. Das älteste moderne Antiepileptikum: Kaliumbromid. 1. Aufl. Offenburg: Davidis Verlag, 1997

${ }^{4}$ Goos M. Bromoderma tuberosum mit Erhöhung der sauren Serumphosphatase Hautarzt 1971; 22: 30-32

${ }^{5}$ Hübner K, Christophers E, Helmer R. Skin bromide content and bromide excretion in bromoderma tuberosum. Arch Dermatol Res 1976; 257: 109-112

${ }^{6}$ Kimmig J. Ursache und Behandlung des Jododerma tuberosum. Hautarzt 1951; 2: $78-79$

${ }^{7}$ Papen G. Bromderm. 1. Fall. Z Hautkr 1972; 47: 258

8 Pfeifle J, Grieben U, Bork K. Bromoderma tuberosum durch antikonvulsive Behandlung mit Kaliumbromid. Hautarzt 1992; 43: $792-794$

${ }_{9}^{9}$ Plewig G, Kligman AM. Akne und Rosazea. 2. Aufl. Berlin, Heidelberg: Springer, 1994

${ }^{10}$ Poser W, Poser S, Echternkamp M. Missbrauch bromhaltiger Schlaf- und Beruhigungsmittel. Dtsch med Wschr 1974; 99: 2489-2497

11 Schmoeckel Ch. Zur Diagnostik und Therapie des Bromoderma tuberosum. Hautarzt 1976; 27: 396 - 398

12 Schöfer H. Zur Therapie des Bromoderma tuberosum mit Furosemid. Z Hautkr 1979; 54: 1019-1026

\section{Dr. G. Wagner}

Chefarzt der Hautklinik

Zentralkrankenhaus Reinkenheide

Postbrookstr. 103

27574 Bremerhaven 